Trauma Berufskrankh 2007 - 9[Suppl 3]: S370-S379

DOI 10.1007/s10039-007-1288-2

Online publiziert: 21. September 2007

(c) Springer Medizin Verlag 2007

T. Tiling $\cdot$ T. Tjardes

Klinik für Unfallchirurgie, Lehrstuhl für Unfallchirurgie/Orthopädie der Universität

Witten/Herdecke, Krankenhaus Merheim, Kliniken der Stadt Köln, Köln

\title{
Der beratende Arzt zwischen Therapiefreiheit und Evidenzzwang
}

dale pharmakologische Behandlung, bis ggf. ein arthroskopischer Eingriff und letztlich ein Gelenkflächenersatz angezeigt sind.

\section{Hintergrund}

Nach oben geschilderten Tatsachen verwundert es nicht, dass bei zunehmendem Kostendruck auch auf die gesetzlichen Unfallversicherungsträger Arbeitshinweise zur Bearbeitung von Arztrechnungen durch den Arbeitskreis Rechnungsprüfung erstellt wurden [15]. Der beratende Arzt der Unfallversicherungsträger wird deshalb in zunehmendem Maß mit der Frage der Notwendigkeit von Behandlungsmaßnahmen im Rahmen der posttraumatischen Arthrosetherapie konfrontiert. Neben der Frage nach dem Kausalzusammenhang zwischen einer Arthrose und dem Unfall ist es nach Beobachtung des Erstautors in den letzten Jahren vor dem Hintergrund der Diskussion um den Stellenwert der Arthroskopie bei der Kniegelenkarthrose (Mosley-Studie) zu einer Zunahme von Beratungsarztanfragen zur Notwendigkeit einer ersten oder auch wiederholten Arthroskopie gekommen. Hinsichtlich der konservativen Therapieoptionen wird insbesondere gehäuft die Frage gestellt, ob die Kosten für Hyaluronsäureinjektionen und Akupunkturtherapie zu übernehmen seien.

\section{Beratender Arzt}

Er ist seit jeher eine feste Einrichtung bei den Haupt- und Bezirksverwaltungen der gesetzlichen Unfallversicherungsträger und der Landesverbände der gesetzlichen Unfallversicherungsträger. Hierauf verweist zuletzt eine Empfehlung des Hauptverbands der Berufsgenossenschaften in einem Rundschreiben VB 1966:

„Bei jeder Berufsgenossenschaft sollte ein beratender Arzt eingestellt werden..."

In den Arbeitshinweisen der Deutschen Gesetzlichen Unfallversicherung (DGUV) zur Bearbeitung von Arztrechnungen wird ausgeführt:

„Bestehen Zweifel an der Notwendigkeit, Zweckmäßigkeit und Wirtschaftlichkeit einer abgerechneten ärztlichen Behandlung (Art, Umfang, Intensität, Behandlungsabstände), sollte der beratende Arzt, bzw. ein für das jeweilige Gebiet kompetenter Facharzt gehört werden. Dieser hat die notwendige Fachkompetenz, zu entscheiden, ob die Grenzen der ärztlichen Therapiefreiheit im Einzelfall überschritten sind." [15]

\section{Therapiefreiheit}

Bei ablehnenden Bescheiden der Unfallversicherungsträger bezüglich der Kostenübernahme bestimmter beantragter Therapien, wie intraartikulärer Hyaluronsäureinjektionen oder Akupunkturtherapie, beruft sich der behandelnde Arzt häufig auf seine Therapiefreiheit. Die Notwendigkeit der von ihm empfohlenen Behandlung wird in der Regel begründet mit:

- der persönlichen Erfahrung,

- dem Verweis auf die Leitlinien der AWMF zur Arthrosetherapie, 
- den in der Gebührenordnung der UV-GOÄ angegebenen Abrechnungsziffern,

- den Indikationsangaben der Industrie,

- Literaturangaben (in Einzelfällen) und

- mit einer höhergradigen evidenzbasierten Literaturrecherche (selten).

In den Arbeitshinweisen der Unfallversicherungsträger zur Bearbeitung von Arztrechnungen wird die Therapiefreiheit des Arztes zwar unterstellt, aber auch eingeschränkt mit der Ausführung:

„Der Arzt hat zwar regelmäßig eine Therapiefreiheit, aber Vergütungen darf er nur für Behandlungen berechnen, die nach den Regeln der ärztlichen Kunst erforderlich und zweckmäßig sind sowie das Gebot der Wirtschaftlichkeit erfüllen ( $\$ 8$ Abs. 1 ÄV). Dieser Grundsatz ist neben dem Grundsatz der Behandlung mit allen geeigneten Mitteln bei der Behandlungsabrechnung der Ärzte zu beachten. Ein Vergütungsanspruch des Arztes ist nur gegeben, wenn die jeweilige ärztliche Leistung medizinisch erforderlich und zugleich zweckmäßig und wirtschaftlich ist. Behandlungsmaßnahmen, die diese Voraussetzung eindeutig nicht erfüllen, sind nicht zu honorieren, d. h. die Rechnung ist ggf. zu korrigieren. Der Arzt ist über die Gründe der Rechnungskürzung zu informieren. “ [15]

\section{Gesetzliche und vertragliche Grundlagen}

Die Unfallversicherungsträger haben bislang die Verpflichtung, „Mit allen geeigneten Mitteln“ Arbeitsunfälle und Berufskrankheiten zu behandeln sowie arbeitsbedingte Gesundheitsgefahren zu verhüten und nach Eintritt eines Arbeitsunfalls oder einer Berufskrankheit die Gesundheit und die Leistungsfähigkeit des Versicherten wiederherzustellen ( $\$ 1$ SGB VII). In \$26 SGB VII wird ausgeführt [5]:

„Erstens: Der Unfallversicherungsträger hat mit allen geeigneten Mitteln möglichst frühzeitig... den durch den Versicherungsfall verursachten Gesundheitsschaden zu beseitigen oder zu bessern, seine Verschlimmerung zu verhindern und seine Folgen zu mildern."

Trauma Berufskrankh 2007 · 9[Suppl 3]: S370-S379 DOI 10.1007/s10039-007-1288-2

(c) Springer Medizin Verlag 2007

T. Tiling $\cdot$ T. Tjardes

Der beratende Arzt zwischen Therapiefreiheit und Evidenzzwang

\section{Zusammenfassung}

Die Kosten für spezielle Formen der Therapie der posttraumatischen Arthrose wie Akupunktur oder Hyaluronsäureinjektionen werden von der DGUV in der Regel nicht übernommen. Es besteht zwar regelmäßig eine Therapiefreiheit für den Arzt, die Vergütung durch die Träger der GUV erfolgt jedoch nur, wenn die Behandlung nach den Regeln der ärztlichen Kunst erforderlich und zweckmäßig ist. Qualität und Wirksamkeit haben dem allgemein anerkannten Stand der medizinischen Erkenntnis zu entsprechen. Die AWMF-Richtlinien stellen nur summarische Beurteilungen der Therapieoptionen dar. Empfehlungen zur Arthrosetherapie werden in den EULAR-Empfehlungen 2003 für

\section{The consultant between freedom of choice in therapy and the requirement for evidence-based medicine}

\section{Abstract}

Special therapies for posttraumatic gonarthrosis, such as acupuncture and hyaluronicacid injections, are not routinely funded by employers' liability insurance associations. Although the physician is generally free to choose the therapeutic approach, therapy is only funded if the treatment is appropriate and necessary according to good clinical practice. Quality and effectivity have to meet current standards of medical knowledge. The AWMF guideline is hardly more than a list summarising potential therapeutic strategies. The EULAR guideline (2003) gives recommendations based on EBM criteria and expert die Kniegelenkarthrose bezüglich der konservativen Behandlung auf EBM-Basis und Expertenmeinung erstellt. Die Studienlage zur konservativen Arthrosetherapie wurde anhand der EBM-Kriterien reevaluiert. Die Literatur und insbesondere die Analysen der Cochrane-Database zeigten, dass Hyaluronsäureinjektionen als wirkungsvolle konservative Therapie der Kniegelenkarthrose erwogen werden müssen, wohingegen die Wirksamkeit vieler anderer Therapiemodalitäten bisher nicht nachgewiesen werden konnte.

\section{Schlüsselwörter}

Arthrose · Erwachsene · Kinder · Richtlinien . EBM

opinions. We reevaluated scientific evidence relating to nonoperative treatment of osteoarthritis of the knee according to EBM criteria, with a special emphasis on the Cochrane Systematic Reviews. These yielded good evidence to show that hyaluronic acid is an option that must be considered for nonoperative treatment of osteoarthritis of the knee, while the effectivity of other approaches has not so far been documented.

\section{Keywords}

Osteoarthritis · Adult · Children · Guidelines . Evidence-based medicine 


\begin{tabular}{|c|c|}
\hline \multirow[t]{3}{*}{ Beratung } & Regelmäßige Übungen zur Beseitigung von Muskeldefiziten \\
\hline & Knieschule \\
\hline & Gewichtskontrolle \\
\hline \multirow[t]{4}{*}{ Peripher wirksame Analgetika } & Antiphlogistika (NSAR) \\
\hline & Steroide (nur lokal) \\
\hline & SYSADO („symptomatic slow acting drugs in osteoarthritis") \\
\hline & Therapeutische Lokalanästhesie \\
\hline \multirow[t]{10}{*}{ Physikalische Maßnahmen } & Physiotherapie \\
\hline & Knieschule \\
\hline & Mobilisierung \\
\hline & Kräftigung \\
\hline & Muskeldehnung \\
\hline & Koordinationsschulung \\
\hline & Thermotherapie \\
\hline & Hydro- und Balneotherapie \\
\hline & Fersenkissen \\
\hline & Bandagen \\
\hline \multirow[t]{4}{*}{ Orthopädietechnik } & Schuhzurichtung (Pufferabsätze, Schuhaußenranderhöhung) \\
\hline & Handstock oder Unterarmgehstützen \\
\hline & Fersenkissen \\
\hline & Bandagen \\
\hline
\end{tabular}

„Viertens: Qualität und Wirksamkeit der Leistung bei Heilbehandlung und Teilhabe haben dem allgemein anerkannten Stand der medizinischen Erkenntnis zu entsprechen und den medizinischen Fortschritt zu berücksichtigen."

„Fünftens: Die Unfallversicherungsträger bestimmen im Einzelfall Art, Umfang und Durchführung der Heilbehandlung und der Leistung zur Teilhabe sowie die Einrichtung, die diese Leistung erbringt nach pflichtgemäßen Ermessen..."

In $\$ 8$ des Ärzteabkommens zwischen Unfallversicherungsträgern und Vertragsärzten (D- und H-Ärzten) wird die ärztliche Behandlung definiert:

„Die ärztliche Behandlung umfasst die Tätigkeiten der Ärzte, die nach den Regeln der ärztlichen Kunst erforderlich und zweckmäßig sind und das Gebot der Wirtschaftlichkeit erfüllen.“

In der Kommentierung von Lauterbach [23] wird ausgeführt, dass die Ermittlung eines allgemein anerkannten Standes der medizinischen Erkenntnis schwierig sei und bei Problemen eine Prüfung im Einzelfall erforderlich ist. Außenseitermethoden sind im Einzelfall möglich, nicht aber ärztliche Behandlungsmaßnahmen, die sich noch in der Erprobungsphase befinden.
Des Weiteren führte Lauterbach [23] aus:

„Die Beschränkungen der KV auf ausreichende, zweckmäßige und wirtschaftliche Maßnahmen, die das Maß des Notwendigen nicht überschreiten dürfen, sind der UV fremd."

Dies gründet sich auf eine unterschiedliche Rechtslage der kassenärztlichen Versorgung nach \$12 SGB V, wo Leistungen im Gegensatz zum Unfallversicherungsrecht nur ausreichend, zweckmäßig und wirtschaftlich sein müssen und das Maß des Notwendigen nicht überschreiten dürfen. Die Übernahme ärztlicher und therapeutischer Maßnahmen nach dem Unfallversicherungsrecht darf somit im Einzelfall nicht allein mit dem Argument zurückgewiesen werden, diese Maßnahmen seien nicht wirtschaftlich. Dies ist von Bedeutung, da im $\$ 8$ des Ärzteabkommens die erforderliche und zweckmäßige ärztliche Tätigkeit quasi gleichwertig neben dem Gebot der Wirtschaftlichkeit aufgeführt wird und damit der Eindruck entsteht, dass das wirtschaftliche Gebot gleichrangigen Charakter hat. Es bekommt jedoch erst Bedeutung, wenn dieselbe Behandlung oder eine gleichwertige Therapie kostengünstiger sind.

Derzeit prüfen die von der Bundesregierung und den Landesregierungen ein- gesetzten Arbeitsgruppen zur Reform der gesetzlichen Unfallversicherung u. a. auch, ob dieser Grundsatz an das Leistungsniveau der gesetzlichen Krankenkasse angepasst werden soll. Damit bleibt abzuwarten, ob sich hier für die Unfallversicherung zukünftig eine neue Rechtslage ergibt.

\section{Leitlinien/Empfehlungen}

\section{Leitlinie Gonarthrose}

Sie wurde von der deutschen Gesellschaft für Orthopädie und orthopädische Chirurgie und dem Berufsverband der Ärzte für Orthopädie zur Therapie der Gonarthrose erstellt. Therapieziele sind:

- die Verminderung der Schmerzen,

- die Verbesserung der Lebensqualität, der Beweglichkeit und der Gehleistung sowie

- die Verzögerung des Fortschreitens der Arthrose.

Die konservativen Therapieoptionen bestehen in Beratung, medikamentöser Therapie, physikalischer Behandlung und Orthopädietechnik (• Tab. 1). Orientierungskriterien für das therapeutische Vorgehen sind:

- der Schmerz,

- das Ausmaß der Arthrose,

- die Therapieresistenz von Maßnahmen,

- das Alter des Patienten,

- die Deformität,

- das Bewegungsausmaß,

- der Leidensdruck und

- die Begleiterkrankungen.

Die erste Stufe der Therapie beinhaltet die zuvor genannten konservativen Verfahren, gefolgt von einer arthroskopischen Operation, der gelenknahen Osteotomie bis hin zum endoprothetischen Ersatz [14].

Eine Bewertung der einzelnen konservativen Maßnahmen bzw. eine stadienbezogene Therapieempfehlung im Sinne eines Stufenschemas der konservativen Therapie der Arthrose finden sich in den Leitlinien nicht. Die von den genannten Fachgesellschaften herausgegebenen Leitlinien beschränken sich auf eine reine 
Aufzählung der ärztlicherseits möglichen Empfehlungen bzw. Verordnungen.

\section{EULAR-Empfehlung Gonarthrose}

Die Empfehlung der Expertenkommission basiert auf einer Literaturanalyse nach den Kriterien der evidenzbasierten Medizin. Insgesamt wurden 545 Publikationen im Rahmen eines Qualitätsscorings der Manuskripte und einer Abschätzung der Therapieeffektivität in die Bewertung eingeschlossen (- Tab. 2). Des Weiteren wurden die Gewichtigkeit der Empfehlungen und das Toxizitätsprofil von Medikamenten bewertet. Schließlich wurde eine Expertenempfehlung im Sinne einer Hilfestellung für die Entwicklung eigener Leitlinien und Algorithmen zur Behandlung der Kniegelenkarthrose erstellt. Ein Therapiealgorithmus wurde bewusst nicht erarbeitet, obwohl die Expertenkommission erkannte, dass ein derart vereinfachter $\mathrm{Zu}$ gang einen größeren Einfluss auf die Entscheidungsfindung vor Ort haben könnte. Es wurde betont, dass es keinen richtigen oder falschen Zugang gibt und dass jeder einzelne Arzt mit seinem individuellen Patienten einen möglichst angemessenen Therapieplan aufstellen muss [22].

Rubin [29] beschrieb einen Algorithmus zur Behandlung des Arthroseknieschmerzes. Neben der nichtmedikamentösen Therapie listete er die Behandlung mit lokalen, oralen und intraartikulären Medikamenten auf und gab für die jeweilige Applikationsart ein Stufenschema an. Für die lokale Therapie empfahl er zunächst Capsaicin, bei unzureichender Verbesserung der Schmerzsymptomatik NSAID und schließlich Lidocain. Bei den oralen Medikamenten sind Acetaminophen oder Glukosamine in der ersten Stufe, bei persistierenden Schmerzen COXII-Inhibitoren oder NSAID mit oder ohne Protonenpumpeninhibitor, dann COX-II-Hemmer mit oder ohne zusätzlichem Tramadol und als Letztes Opioide indiziert. Intraartikulär sollten zunächst Kortikosteroide und bei weiter bestehenden Schmerzen Hyaluronsäure appliziert werden. Empfehlungen, welche Therapieoptionen ggf. kombiniert werden können oder sollten, werden nicht gegeben.

Letztlich weist die EULAR-Empfehlung darauf hin, dass die Behandlung in- dividuell auf den jeweiligen Patienten abgestimmt erfolgen sollte.

\section{Evidenzbasierte Literaturanalyse}

Bis Februar 2007 erfolgte eine systematische Literatursuche in Medline zur konservativen Therapie der Arthrose und speziell der Kniegelenkarthrose. Eingeschlossen in die Literaturauswertung wurden nur Studien der Evidenzgrade Ia-IIb (CEBM, [30]). Ausgeschlossen wurden Fall-Kontroll-Studien, Fallberichte und klinische Erfahrungsberichte (Evidenzgrade III-V). Insbesondere wurden die Studien zur Osteoarthrosetherapie der Cochrane-Database herangezogen. Im Fall des Vorhandenseins eines Reviews $\mathrm{zu}$ einer bestimmten Arthrosetherapie wurden das Ergebnis und die Bewertung durch die Cochrane-Collaboration übernommen und im Einzelfall durch zeitlich neuere Publikationen der Evidenzgrade III ersetzt.

In der nachfolgenden Auswertung wurden für die jeweilige Therapie die Art und die Anzahl der Studien bzw. die Studienpopulation, deren Evidenzgrad und die Ergebnisse der Studien bewertet. Ergänzt wurde die Bewertung der einzelnen Therapiemaßnahmen durch eine persönliche Beurteilung deren Wirksamkeit entsprechend den Vorgaben des \$26 SGB VII unter Hinweis auf die obigen Ausführungen.

\section{Nichtmedikamentöse Therapie}

Eine Übersicht mit Angaben zur Anzahl der Studien, dem Evidenzgrad, den Ergebnissen und der eigenen Bewertung der Wirksamkeit findet sich in $\mathbf{0}$ Tab. 3. Im Einzelnen wurden die nachfolgenden nicht medikamentösen Therapien analysiert.

\section{Selbstmanagement}

Aus 780 analysierten Studien wurden 14 zur Osteoarthrose bewertet. Die Metaanalyse (Evidenzgrad Ia) ergab, dass Selbstmanagementprogramme keinen Effekt auf den Schmerz und die Funktion bei der Osteoarthrose haben [13]. In einer weiteren Metaanalyse von Devos-Comby et al. [16] wurden 16 kontrollierte Studien zu Eigenübungen und Selbstmanagement untersucht. Sie fanden einen mäßi-
Tab. 2 EULAR-Empfehlungen 2003.

(Mod. nach [22])

1. Das optimale Management benötigt eine Kombination aus nichtmedikamentöser Therapie und Medikamenten.

2. Die Behandlung sollte abgestimmt sein auf: Knierisikofaktoren, generelle Risikofaktoren, Schmerzstärke und Behinderung, Erguss sowie Arthrosegrad.

3. Nichtmedikamentöse Therapie sollte beinhalten: Erziehung, Übungen, Hilfsmittel und Gewichtsreduktion.

4. Paracetamol zuerst und bei Wirksamkeit bevorzugtes Langzeitmedikament

5. Lokale Medikamente (NSAID, Capsaicin) sind wirksam.

6. Opioide sind nützlich bei Paracetamolunwirksamkeit oder NSAID-Unverträglichkeit.

7. SYSADOA (Glukosamin, Chondroitinsulfat, ASIS, Hyaluronsäure) haben symptomatischen Effekt.

8. Intraartikuläre Injektionen von lang wirkenden Kortikosteroiden sind indiziert bei extremen Schmerzen besonders in Kombination mit Ergussbildung.

gen, aber klinisch relevanten psychologischen Effekt, dahingehend dass die Patienten sich besser fühlten.

Diese Ergebnisse wurden in einer weiteren neuen Studie bestätigt [10]. Durch die Teilnahme an einem Selbsthilfeprogramm konnte ohne eine Zunahme der Schmerzen und Behinderung die Dosis von nichtsteroidalen Antiphlogistika reduziert werden [24].

\section{Gymnastik}

Die Analyse von 17 randomisierten, kontrollierten Studien (Evidenzgrad Ia) ergab eine Verbesserung durch Gymnastik sowohl für den Schmerz als auch für die Kniegelenkfunktion. Es fand sich kein Unterschied, ob die Behandlung als Einzeloder Gruppentherapie durchgeführt wurde [19]. Anhand dieser Studien konnte jedoch nicht festgestellt werden, welches Physiotherapieprogramm die besten Ergebnisse erzielt, in welcher Frequenz trainiert werden sollte, mit welcher Intensität und für wie lange es durchgeführt werden sollte.

In einer neueren kontrollierten Studie wurde ein Physiotherapieprogramm in einer randomisierten, doppelblind kontrollierten Studie an 140 Patienten überprüft. 


\section{Konservative Arthrosebehandlung}

\begin{tabular}{|c|c|c|c|c|c|}
\hline \multirow[t]{2}{*}{ Therapie } & \multirow[t]{2}{*}{ Studie(n) } & \multirow[t]{2}{*}{ Anzahl } & \multirow{2}{*}{ 总 } & \multicolumn{2}{|l|}{ Ergebnisse } \\
\hline & & & & Cochrane-Bewertung & $\begin{array}{l}\text { Eigene Beurteilung } \\
\text { der Wirksamkeit } \\
\text { (§26 SGB VII) }\end{array}$ \\
\hline Selbstmanagement & Metaanalysen (2) & $\begin{array}{l}14 \mathrm{RCT} \\
16 \mathrm{RCT}\end{array}$ & la & $\begin{array}{l}\text { Kein Effekt auf Schmerz und Funktion } \\
\text { Mäßig positiver psychologischer Effekt }\end{array}$ & Nicht wirksam \\
\hline Gymnastik & Metaanalyse & $\begin{array}{l}17 \mathrm{RCT} \\
(\mathrm{n}=2562)\end{array}$ & la & $\begin{array}{l}\text { Verbesserung für Schmerz und Funktion } \\
\text { Kein Unterschied für Gruppen- vs. Einzeltherapie }\end{array}$ & Wirksam \\
\hline $\begin{array}{l}\text { Brace und } \\
\text { Schuheinlage }\end{array}$ & $\begin{array}{l}\text { Mit vs. ohne Brace/ } \\
\text { Orthese/Einlage }\end{array}$ & $\begin{array}{l}4 \mathrm{RCT} \\
(\mathrm{n}=444)\end{array}$ & $\mathrm{lb}$ & $\begin{array}{l}\text { Nur mediale Gonarthrose } \\
\text { Positiver Effekt bei zusätzlicher medikamentöser } \\
\text { Therapie: verminderte NSAID-Einnahme }\end{array}$ & Wirksam \\
\hline Kryotherapie & $\begin{array}{l}\text { Eismassage } \\
\text { Kältekissen } \\
\text { Wärmekissen }\end{array}$ & $\begin{array}{l}\text { 3 Studien } \\
(\mathrm{n}=179)\end{array}$ & $\mathrm{lb}$ & $\begin{array}{l}\text { Verbesserte Quadrizepskraft, Beugung und Funktion } \\
\text { Verbessertes Ödem } \\
\text { Wärmekissen: kein Effekt auf Schwellung } \\
\text { Kühltherapie: keine Schmerzreduktion }\end{array}$ & Nicht zweckmäßig \\
\hline $\begin{array}{l}\text { Therapeutischer } \\
\text { Ultraschall }\end{array}$ & $\begin{array}{l}\text { Ultraschall vs. Placebo } \\
\text { Ultraschall vs. } \\
\text { galvanischer Strom } \\
\text { Ultraschall vs. Kurzwelle }\end{array}$ & $\begin{array}{l}3 \text { Studien } \\
(n=294)\end{array}$ & $\mathrm{lb}$ & $\begin{array}{l}\text { Alle } 3 \text { Studien zeigten keinen Effekt (Schmerz, ,gait } \\
\text { velocity“) }\end{array}$ & Unwirksam \\
\hline $\begin{array}{l}\text { Low-Level-Laser- } \\
\text { Therapie }\end{array}$ & Laser vs. kein Laser & $\begin{array}{l}7 \mathrm{RCT} \\
(\mathrm{n}=184)\end{array}$ & la & $\begin{array}{l}\text { Kein Benefit für Schmerz im 1. Monat } \\
3 \text { Studien eher positiver Effekt } \\
3 \text { Studien kein Effekt } \\
1 \text { Studie Schmerzminderung (keine Schmerzmessung) }\end{array}$ & $\begin{array}{l}\text { Wirksamkeit nicht } \\
\text { nachgewiesen }\end{array}$ \\
\hline \multirow[t]{2}{*}{$\begin{array}{l}\text { Akupunktur/ } \\
\text { Elektroakupunktur } \\
\text { (rheumatoide } \\
\text { Arthritis) }\end{array}$} & $\begin{array}{l}\text { Akupunktur vs. Placebo } \\
\text { Elektroakupunktur } \\
\text { vs. Placebo } \\
\text { Traditionelle chinesische } \\
\text { Akupunktur vs. Sham- } \\
\text { Akupunktur ([31]) }\end{array}$ & $\begin{array}{l}3 \text { Studien } \\
(\mathrm{n}=84 / 1007)\end{array}$ & $\mathrm{lb}$ & $\begin{array}{l}\text { Studie 1: keine Änderung bezüglich BKS, CRP, VHS, gen. } \\
\text { „health question“, Steifheit, Schwellung Anzahl betrof- } \\
\text { fener Gelenke, „, disease activity scale" } \\
\text { Studie 2: Schmerzreduktion nach } 24 \text { h/4 Monaten, } \\
\text { schlechtes Design, zu kleine Population } \\
\text { Traditionelle chinesische Akupunktur gleich wirksam wie } \\
\text { Sham-Akupunktur, könnte Placebowirkung sein }\end{array}$ & $\begin{array}{l}\text { Wirksamkeit nicht } \\
\text { nachgewiesen }\end{array}$ \\
\hline & Metaanalyse & $7 \mathrm{RCT}(\mathrm{n}=393)$ & la & & \\
\hline TENS & TENS vs. kein TENS & $\begin{array}{l}6 \text { Studien } \\
(n=148 / 146)\end{array}$ & la & Verbesserung der Schmerzen und Steifheit & Wirksam \\
\hline $\begin{array}{l}\text { Elektromagnetische } \\
\text { Feldtherapie }\end{array}$ & $\begin{array}{l}\text { Elektromagnetische } \\
\text { Feldtherapie vs. keine } \\
\text { elektromagnetische } \\
\text { Feldtherapie }\end{array}$ & $3 \mathrm{RCT}(\mathrm{n}=259)$ & la & $\begin{array}{l}\text { "clinical benefit" in } 13-23 \% \\
\text { Studien mit größerer Patientenzahl zur Beurteilung der } \\
\text { klinischen Relevanz nötig }\end{array}$ & $\begin{array}{l}\text { Wirksamkeit nicht } \\
\text { nachgewiesen }\end{array}$ \\
\hline
\end{tabular}

Hier ergab die 12-wöchige Physiotherapie mit nachfolgendem Selbstmanagementprogramm gegenüber einer Placebotherapie mit nicht wirksamem Gel und Placeboultraschall eine Verbesserung des Schmerzes und der Behinderung in beiden Gruppen. Ein signifikanter Unterschied zwischen der Physiotherapie- und der Placebogruppe konnte nicht festgestellt werden [3].

Brosseau et al. [6] konnten zeigen, dass eine bloße Erhöhung der Intensität bei der Aerobicgymnastik keinen Unterschied in der Funktion, dem Grad der Behinderung und im Schmerz ergibt.

\section{Brace- und Schuheinlage}

In 4 kontrollierten Studien (Evidenzgrad Ib) wurden ein valgisierender Knie-Brace,
Bandage und lateral erhöhte Einlagenversorgung für die Varusgonarthrose getestet. Alle Hilfsmittel ergaben eine Verbesserung im Funktionstest (WOMAC) und eine geringe Reduktion des Antiphlogistikabedarfs [9].

\section{Thermotherapie}

In 3 kontrollierten Studien mit 179 Patienten wurde die Wirksamkeit von Eismassage, Kälte- und Wärmekissen untersucht (Evidenzgrad Ib). Eismassage konnte die Beweglichkeit und die Quadrizepskraft verbessern, das Kältekissen verbesserte die Schwellneigung. Wärmekissen hatten keinen Effekt auf die Knieschwellung. Die Kryotherapie führte nicht zur Schmerzreduktion [7].

\section{Ultraschall und Elektrotherapie}

In 3 kontrollierten Studien wurde Ultraschall gegen Placebo, galvanischen Strom oder Kurzwellenbestrahlung mit insgesamt 294 Patienten untersucht (Evidenzgrad Ib). Alle 3 Studien zeigten keinen Therapieeffekt [28].

\section{Lasertherapie}

Sie wurde in 7 kontrollierten Studien $(n=184)$ gegen Placebolaser $(n=161)$ getestet (Evidenzgrad Ia). 3 Studien ergaben keinen Unterschied bei den Schmerzmessungen, während 2 über einen sehr guten analgetischen Effekt der Lasertherapie berichteten. In einer weiteren Studie ohne Schmerzmessung wurde von den $\mathrm{Pa}$ tienten eine Schmerzverbesserung durch die Lasertherapie angegeben. Eine Studie 
zeigte eine Verbesserung der Kniegelenkbeweglichkeit.

Die widersprüchlichen Ergebnisse machen weitere kontrollierte Studien erforderlich [8].

\section{Akupunktur und Elektroakupunktur}

2 Studien mit insgesamt 84 Patienten untersuchten Akupunktur, bzw. Elektroakupunktur gegen Scheinakupunktur bei Patienten mit rheumatoider Arthritis (Evidenzgrad Ib). In einer Studie ergaben sich keine signifikanten Veränderungen, während in der 2. eine Schmerzminderung durch die Akupunktur angegeben wurde, wobei diese Studie jedoch eine geringe Qualität aufwies und der Effekt der Schmerzlinderung überschätzt wurde [11].

In einer ersten Metaanalyse ergaben 4 von 7 kontrollierten Studien bei insgesamt 393 Patienten einen statistisch signifikanten Effekt der Akupunktur gegenüber einer konservativen Behandlung allein oder Scheinakupunktur (Evidenzgrad Ia), wobei die meisten Studien ein schlechtes Design und kurze Follow-upZeiten aufwiesen [18].

In einer weiteren 3-armigen kontrollierten Studie aus Deutschland, die nicht in die vorgenannte Metaanalyse eingegangen ist, wurden 1007 Patienten mit einer Arthrose eingeschlossen die 40 Jahre oder älter waren. In einer Kohortenstudie wurden 3 Arme verglichen. Alle Patienten erhielten 6 Physiotherapiesitzungen und Diclophenac sowie in einer Gruppe ggf. Rofecoxib. Zusätzlich erhielt die erste Gruppe 10 und ggf. 5 weitere Male traditionelle chinesische Akupunktur, die 2. Gruppe eine Scheinakupunktur und die 3. Gruppe keine Akupunktur. Die Patienten wurden im Verhältnis 1:1:1 blockrandomisiert a 6 Patienten. Eine Verblindung erfolgte nicht. Die Gruppen mit Akupunktur bzw. Scheinakupunktur erzielten eine größere Verbesserung im WOMACScore nach 26 Wochen. Es fand sich jedoch keine statistische Signifikanzdifferenz zwischen der traditionellen chinesischen Akupunktur und der Scheinakupunktur. Eine Kontrollgruppe mit Placebotherapie gab es in dieser Studie nicht (Evidenzgrad Ib). Die Autoren schlossen aus ihrer Studie, dass Scheinakupunktur und traditionelle Akupunktur der allei- nigen konservativen Therapie mit Physiotherapie und Antiphlogistika überlegen sind. Wegen der fehlenden Verblindung erlaubt diese Studie aber keine Aussage darüber, ob die nachgewiesene Wirkung ein Placeboeffekt, eine Auswirkung der Kontakte mit dem Akupunkteur oder ein psychologischer Effekt der Nadelsetzungen ist [31].

In der Gesamtbeurteilung all dieser kontrollierten Studien scheint damit nicht der Nachweis erbracht zu sein, dass eine Akupunkturtherapie wirksam ist. Eine verblindete kontrollierte Placebostudie ist zum Erreichen einer besseren Evidenz erforderlich.

\section{Transkutane elektrische Nervenstimulation (TENS)}

In die Analyse wurden 7 Studien eingeschlossen, wobei in 6 Studien die klassische und in einer Studie eine akupunkturähnliche TENS-Therapie bei $292 \mathrm{~Pa}$ tienten analysiert wurden. Es handelte sich um 3 Crossover- und 4 randomisierte, placebokontrollierte Studien. TENSTherapie war bezüglich der Schmerzlinderung und Bewegungsverbesserung bei der Kniegelenkarthrosebehandlung signifikant besser [27].

\section{Elektromagnetische Feldtherapie}

3 kontrollierte Studien mit 359 Patienten wurden in die Auswertung einbezogen. Die elektromagnetische Stimulationstherapie hatte zwar einen statistisch signifikanten, gering bis mäßig positiven Effekt auf die Verbesserung der kniegelenkarthrosebedingten Schmerzsymptomatik, aber diese positive Wirkung erschien fraglich bzw. klinisch nicht relevant. Zur Beurteilung der klinischen Relevanz des Therapieeffekts sind Studien mit größeren Patientenzahlen erforderlich [21]. In einer weiteren Metaanalyse aus 2006 wurden 5 kontrollierte Studien bezüglich der Wirksamkeit der gepulsten elektromagnetischen Feldtherapie analysiert. Danach bewirkt diese keine signifikante Schmerzreduktion [25].

\section{Medikamentöse Therapie}

Eine Zusammenfassung der Metaanalysen über die unterschiedlichen medikamentösen Therapieformen mit Angabe des Evidenzgrads und des Ergebnisses der Bewertung sowie der eigenen Beurteilung der Wirksamkeit findet sich in $\square$ Tab. 4. Im Einzelnen wurden in den Metaanalysen die nachfolgenden Medikamente untersucht.

\section{Acetaminophen}

In einer Metaanalyse wurden 17 randomisierte kontrollierte Studien mit 2562 Patienten analysiert, 7 Studien verglichen Acetaminophen mit Placebo und 10 Studien mit nichtsteroidalen Antiphlogistika. Bei einem vergleichbaren Sicherheitsprofil war Acetaminophen der Placebotherapie überlegen. Die Schmerzen wurden signifikant reduziert. Ein sicherer Unterschied in der Sicherheit der Acetaminophen- und NSAID-Therapie konnte nicht gefunden werden (Evidenzgrad Ia). NSAID-Patienten hatten gegenüber der Acetaminophengruppe weniger Schmerzen und Bewegungseinschränkung und eine bessere physikalische Funktion. Bei Einnahme von traditionellen NSAID wie Ibuprofen oder Naproxin hatte diese Gruppe jedoch mehr abdominelle Probleme als Patienten mit Acetominopheneinnahme. Damit ist Acetominophen in der Arthrosetherapie als wirksam zu bewerten [34].

\section{Lokale NSAID}

2 Metaanalysen beurteilten die Wirksamkeit lokaler nichtsteroidaler antiphlogistischer Medikamente gegen Placebo bzw. gegen Diclophenac oral (Evidenzgrad Ia). In die Metaanalyse von Biswal et al. [5] wurden 4 placebokontrollierte Studien mit insgesamt 811 Patienten eingeschlossen. In die Metaanalyse von Toweed [32] wurden 3 placebokontrollierte Studien und in einer Studie ein lokales Antiphlogistikum gegen orale Diclophenacverordnung bei insgesamt 1412 Patienten analysiert. Beide Metaanalysen belegten, dass die lokale Anwendung nichtsteroidaler Antiphlogistika sowohl bezüglich der Schmerzreduktion als auch hinsichtlich der Gelenk- und der Gesamtfunktion wirksam ist. Als unerwünschte Nebenwirkungen traten Hautreaktionen auf. In einer der kontrollierten Studien (lokale vs. orale NSAID-Therapie) waren die Ergebnisse vergleichbar, wobei die Patienten mit der oralen Diclophenaceinnahme eine höhere Rate an 


\section{Konservative Arthrosebehandlung}

\begin{tabular}{|c|c|c|c|c|c|}
\hline \multirow[t]{2}{*}{ Therapie } & \multirow[t]{2}{*}{ Studie(n) } & \multirow[t]{2}{*}{ Anzahl } & \multirow{2}{*}{ 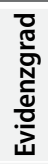 } & \multicolumn{2}{|l|}{ Ergebnisse } \\
\hline & & & & Cochrane-Bewertung & $\begin{array}{l}\text { Eigene Beurteilung } \\
\text { der Wirksamkeit } \\
\text { (§26 SGB VII) }\end{array}$ \\
\hline \multirow{2}{*}{$\begin{array}{l}\text { Acetaminophen } \\
\text { (Paracetamol) }\end{array}$} & Acetaminophen vs. Placebo & $7 \mathrm{RCT}$ & \multirow[t]{2}{*}{ la } & 5 Studien: Acetaminophen besser als Placebo & \multirow[t]{2}{*}{ Wirksam } \\
\hline & Acetaminophen vs. NSAID & $\begin{array}{l}10 \mathrm{RCT} \\
(\mathrm{n}=5986)\end{array}$ & & $\begin{array}{l}2 \text { Studien: kein Unterschied, kein Effekt auf } \\
\text { Steifheit, Bewegung } \\
\text { Acetaminophen weniger wirksam als NSAID } \\
\text { Acetaminophen und NSAID gleich sicher, } \\
\text { aber } 13 \% \text { vs. } 19 \% \text { weniger adverse Events } \\
\text { Schmerzreduktion durch NSAID gegenüber } \\
\text { Acetaminophen } 6 / 100 \\
\text { NSAID: weniger Schmerz, weniger Steifheit, } \\
\text { bessere Bewegung }\end{array}$ & \\
\hline Lokale NSAID & 2 Metaanalysen $(n=811 / 1412)$ & $\mathrm{RCT}$ & la & Lokale NSAID sind wirksam & Wirksam \\
\hline NSAID & Metaanalyse & $16 \mathrm{RCT}$ & la & $\begin{array}{l}\text { Kein wissenschaftlich gesicherter Unter- } \\
\text { schied verschiedener NSAID wegen Mängeln } \\
\text { (Irrtümern im Studiendesign) }\end{array}$ & Wirksam \\
\hline Tramadol und Opioide & $\begin{array}{l}\text { Metaanalyse } \\
\text { Tramadol vs. Placebo } \\
\text { Tramadol vs. NSAID }\end{array}$ & $\begin{array}{l}11 \mathrm{RCT} \\
(\mathrm{n}=1019+920)\end{array}$ & la & $\begin{array}{l}\text { Signifikante Schmerzreduktion } \\
1 \text { von } 8 \text { Patienten Medikament abgesetzt } \\
\text { wegen Nebenwirkungen }\end{array}$ & Wirksam \\
\hline $\begin{array}{l}\text { Kortison (intraarti- } \\
\text { kulär) }\end{array}$ & Metaanalyse & $\begin{array}{l}28 \mathrm{RCT} \\
(\mathrm{n}=1973)\end{array}$ & la & $\begin{array}{l}\text { Kortikosteroide sind effektiv: für Schmerz } \\
\text { und „global assessment“ für 1-3 Wochen, } \\
\text { nach 4-24 Wochen kein Effekt } \\
\text { Kein Unterschied Kortikoide vs. Hyaluron- } \\
\text { säure nach 1-4 Wochen, Hyaluronsäure nach } \\
\text { 5-13 Wochen effektiver }\end{array}$ & $\begin{array}{l}\text { Wirksam, aber nicht } \\
\text { zweckmäßig }\end{array}$ \\
\hline Glukosamine & Metaanalyse & $\begin{array}{l}20 \mathrm{RCT} \\
(\mathrm{n}=2170)\end{array}$ & la & $\begin{array}{l}\text { Positiver Effekt bezüglich Schmerz (13/100 } \\
\text { vs. Placebo) }\end{array}$ & $\begin{array}{l}\text { Nur Rotta- } \\
\text { Präparationen } \\
\text { schmerzeffektiv, aber } \\
\text { nicht im WOMAC }\end{array}$ \\
\hline $\begin{array}{l}\text { Hyaluronsäure und } \\
\text { Hyaluronderivate }\end{array}$ & Metaanalyse & $76 \mathrm{RCT}$ & la & $\begin{array}{l}5-13 \text { Wochen nach intraartikulärer Injektion } \\
\text { bei } 28-54 \% \text { Verbesserung der Schmerzen, } \\
\text { bei } 9-32 \% \text { Verbesserung der Funktion }\end{array}$ & Wirksam \\
\hline
\end{tabular}

Nebenwirkungen im Bereich des Gastrointestinaltrakts aufwiesen [35].

\section{Orale NSAID}

In die Analyse der Cochrane-Database wurden 16 randomisierte Studien eingeschlossen. In 11 wurde Ethodolac, in 3 wurden Naproxem und Diclophenac und in je 1 Indometacin und Nabumethon untersucht. Bei einem Evidenzgrad Ia sind nichtsteroidale Antiphlogistika wirksam für die Schmerzreduktion und Verbesserung der Funktion. Ein wissenschaftlich gesicherter Unterschied der Wirksamkeit der unterschiedlichen Antiphlogistika konnte wegen Mängeln im Studiendesign nicht festgestellt werden [36].

In einer weiteren Metaanalyse wurden 26 placebokontrollierte Studien bewertet, in denen Diclophenac, Ibuprofen, Naproxin, Ninesulide, Nabumethon, Paracetamol, Celecoxit und Arthotec im Ver- gleich zu Rofecoxit untersucht wurden. Vioxx $^{\circledast}$ (Wirkstoff Rofecoxit) wurde 2004 wegen eines möglicherweise erhöhten Risikos für Herzattacken und Schlaganfälle vom Markt genommen. Aus diesen Studien ist jedoch zu erkennen, dass die Wirksamkeit der verschiedenen nichtsteroidalen Antiphlogistika, bei ähnlichen Nebenwirkungen bezüglich abdomineller Probleme, vergleichbar ist. Ibuprofen ist mit einer höheren Rate an Magenproblemen behaftet, während Arthotec eine höhere Durchfallrate gegenüber Rofecoxit zeigt. Die Autoren folgerten, dass neben der Dosierung eine erhebliche individuelle Komponente besteht, die den Therapieerfolg oder das Auftreten von Nebenwirkungen mitbestimmt. Der Effekt und die Sicherheit der COX-II-Hemmer sind noch nicht abschließend zu beurteilen [20].

\section{Tramadol und Opioide}

Ausgewertet wurden 11 kontrollierte Studien mit 1019 Patienten, die Tramadol oder Tramadol und Paracetamol erhielten, und 920 Patienten, die Placebo oder eine aktive Kontrolle erhielten. Tramadol reduziert die Schmerzen bei der Osteoarthrose wirksam, hat jedoch ein 2,3fach erhöhtes Risiko, geringe Nebenwirkungen, und 2,6-faches Risiko, schwerere Nebenwirkungen als das Placebo zu bewirken (Evidenzgrad Ia). 1 von 8 Patienten beendete wegen dieser Nebenwirkungen die Tramadoleinnahme. Rückschlüsse auf die Wirksamkeit im Vergleich zu anderen Medikamentenbehandlungen sind wegen der limitierten Anzahl von Studien nicht möglich.

Tramadol ist wirksam in Bezug auf die Schmerzen und die Beweglichkeit bei der Osteoarthrose [12]. 


\section{Kortison intraartikulär}

In einer Metaanalyse wurden 28 kontrollierte Studien zur Wirksamkeit intraartikulärer Kortikosteroidinjektion gegen Placebo, gegen Hyaluronsäure, gegen Lavage und andere Kortikosteroide bei 1973 Patienten untersucht und beurteilt. Kortikoide sind effektiv in der Schmerzreduktion und der Verbesserung der globalen Funktion für einen Zeitraum von 1-3 Wochen nach der Injektion, aber nicht darüber hinaus (Evidenzgrad Ia). Ein Unterschied der Wirksamkeit von Kortikoiden gegenüber Hyaluronsäure konnte bis zu 4 Wochen nicht erkannt werden. Ab der 4. Woche war die Injektion von Hyaluronsäure bis zu einem Nachuntersuchungszeitraum von 5-13 Wochen jedoch effektiver [1].

In dieser Metaanalyse wurden keine Angaben hinsichtlich der Wirksamkeit wiederholter Kortikosteroidinjektionen und der möglichen und tatsächlichen $\mathrm{Ne}$ benwirkungen gemacht. Eine NutzenRisiko-Bewertung, sei es zur Frage einer steroidinduzierten Osteonekrose, der Progression der Osteoarthrose, der Verschlechterung einer diabetischen Stoffwechsellage oder dem Infektrisiko wurde nicht vorgenommen.

In der eigenen Bewertung kommen wir zu dem Ergebnis, dass eine intraartikuläre Kortisoninjektion wirksam ist, aber die Zweckmäßigkeit nicht ausreichend geprüft ist. Insbesondere liegt kein systematischer Vergleich der Nebenwirkungsprofile der verschiedenen Therapieoptionen (nichtsteroidale Antiphlogistika, Analgetika und Glukosamine) vor, sodass in der Nutzen-Risiko-Abwägung intraartikuläre Kortisoninjektionen als nicht zweckmäßig beurteilt werden müssen. Darüber hinaus scheinen Hyaluronsäureinjektionen in der Langzeitwirkung den Kortikoiden überlegen zu sein.

\section{Glukosamine}

In einer Metaanalyse (Evidenzgrad Ia) zur Bewertung der Wirksamkeit von Glukosaminen wurden 20 randomisierte Studien mit 2170 Patienten einbezogen. Gegenüber Placebo besteht ein gesicherter Effekt in der Schmerzreduktion, wobei aber nur so genannte Rotta-Präparationen schmerzeffektiv sind. In 2 kontrollierten Studien zeigten Glukosamine im Vergleich zu
NSAID eine bessere und in 2 Studien eine vergleichbare Wirksamkeit. In 2 kontrollierten Studien wurde eine Verlangsamung der radiologischen Progression der Osteoarthrose des Kniegelenks über einen Beobachtungszeitraum von 3 Jahren beschrieben. Glukosamine sind genauso sicher wie Placebo bezüglich der $\mathrm{Ne}$ benwirkungen. Eine funktionelle Verbesserung (WOMAC) konnte jedoch nicht nachgewiesen werden. Die Autoren betonten jedoch, dass eine abschließende Beurteilung der Wirksamkeit nur bedingt möglich ist, da bei Einbeziehung nur der besten Studien der Nachweis eines Effekts bezüglich der Schmerzen nicht mehr erbracht werden kann [33].

In der eigenen Bewertung bestehen daher Zweifel bezüglich der Wirksamkeit von Glukosaminen, da jetzt aktuelle und methodisch zuverlässigere Studien vorliegen, die gegenüber der ersten CochraneAnalyse von 1999 nicht mehr einheitlich einen positiven Schmerzeffekt nachweisen konnten.

\section{Hyaluronsäure und Hyaluronsäurederivate}

In die aktuelle Metaanalyse zur Wirksamkeit von Hyaluronsäure und Hylan wurden 40 Studien eingeschlossen. In 10 Studien wurde gegen intraartikuläre Kortikosteroide, in 6 gegen NSAID, in 3 gegen Physiotherapie, in 2 gegen allgemeine Übungen, gegen eine Arthroskopie oder konventionelle Therapie getestet. 15 Studien verglichen unterschiedliche Hyaluronsäure-/Hylanpräparate miteinander. Nach 5-13 Wochen konnte ein positiver Schmerzeffekt verzeichnet werden. Die Effektivität war vergleichbar mit nichtsteroidalen Antiphlogistika. Der Langzeiteffekt im Vergleich zu intraartikulären Kortikosteroidinjektionen war jedoch deutlich besser, bei gleichzeitig geringerer $\mathrm{Ne}$ benwirkungsrate (Evidenzgrad Ia).

In der Zusammenfassung folgerten die Autoren der aktuellen Metaanalyse, dass Hyaluronsäureinjektionen effektiv sind bezüglich der Verminderung des Schmerzes, der Verbesserung der Funktion und der Verbesserung der Gesamtfunktion [4].

Bemerkenswert ist die Tatsache, dass sich bei Vergleichsstudien von Hylan gegen NSAID eine vergleichbare Effektivität ergab, allerdings mit mehr systemischen Nebenwirkungen in der NSAID-Gruppe. Bezogen auf die Sicherheit ergab sich ein vergleichbares oder gering besseres Ergebnisse für Hylan. In einer weiteren Vergleichsstudie von Orthovisc ${ }^{\varpi}$ mit Physiotherapie gegen Physiotherapie allein ergab sich kein Wirksamkeitsunterschied. Gegenüber nichtinvasiven Therapien ergab sich eine höhere Rate an lokalen $\mathrm{Ne}$ benwirkungen bei der Hyaluronsäureinjektionsgruppe. Patienten mit einer höhergradigen Arthrose scheinen weniger auf die Hyaluronsäuretherapie anzusprechen [4].

In der eigenen Bewertung kommen wir in der Beurteilung der Wirksamkeit aufgrund der aktuellen Cochrane-Metaanalyse aus 2006 zur der Aussage, dass Hyaluronsäure und ihre Derivate wirksam sind. Es gelten jedoch die gleichen Einschränkungen wie für die Kortisontherapie bezüglich der Notwendigkeit einer Nutzen-Risiko-Abwägung. Der Schmerz lindernde Effekt der Hyaluronsäuretherapie scheint im Einzelfall nur bis zu einem halben Jahr anzuhalten. Es sind jeweils mehrere Injektionen (3-5) erforderlich, durch die es im Einzelfall zu einer Gelenkinfektion kommen kann, was für eine evtl. erforderlich werdende operative Intervention (insbesondere Prothesenimplantation) prognostisch ungünstig ist. Auf diese Problematik verweist auch der aktuelle systematische Übersichtsartikel von Divine et al. [17], die 5 Metaanalysen auswerteten. Alle stützen die Annahme, dass in einem selektierten Patientengut mit einer Osteoarthrose die Hyaluronsäuretherapie effektiv ist. In jedem Fall müssen die möglichen Risiken und die Kosten gegen den positiven Effekt der Therapie sorgfältig abgewogen werden.

\section{Diskussion}

Die posttraumatische Osteoarthrose stellt für die gesetzlichen Unfallversicherungsträger eine relevante Kostenbelastung bezüglich der Gewährung von Renten, der Verletztengeldzahlung im Rahmen einer Wiedererkrankung und der ärztlich verordneten Therapiekosten dar. Zunehmend wird daher der beratende Arzt zur Frage eingeschaltet, ob insbesondere Akupunkturtherapien und Hyaluronsäurein- 
jektionen seitens der Unfallversicherungsträger zu übernehmen seien, da der Unfallversicherungsträger im Einzelfall Art, Umfang und Durchführung der Behandlung bestimmt. Hierbei müssen Qualität und Wirksamkeit der Leistung bei Heilbehandlung dem allgemeinen anerkannten Stand der medizinischen Erkenntnis entsprechen. Weitgehend anerkannt ist in Deutschland ärztlicherseits, dass eine solche Beurteilung - so weit vorhanden - auf dem besten erreichbaren wissenschaftlichen Evidenzgrad unter Einbeziehung der individuellen Patientensituation erfolgen sollte.

Die Leitlinie „Gonarthrose“ der Deutschen Gesellschaft für Orthopädie und orthopädische Chirurgie und des Berufsverbands der Ärzte der Orthopädie zur Gonarthrose beschränkt sich auf eine Auflistung der neben der reinen Beratung für möglich erachteten Behandlungsmaßnahmen, ohne dass eine evidenzbasierte Bewertung angegeben wird. Die EULAREmpfehlung aus 2003 empfiehlt für die Gonarthrosetherapie evidenzbasiert eine Kombination aus nichtmedikamentöser und medikamentöser Therapie in Abhängigkeit von den Risikofaktoren, der Schmerzstärke, der Behinderung, dem Ergussausmaß und dem Arthrosegrad. Als nichtmedikamentöse Behandlung werden Übungen empfohlen, wobei evidenzbasiert Eigenmanagementprogramme wohl keinen Effekt auf den Schmerz und die Funktion des geschädigten Gelenks sowie die Arthrosekrankheit selbst haben.

Wirksam ist jedoch eine Physiotherapie. Ihre Verordnung bei einer posttraumatischen Arthrose wird kostenmäßig regelhaft seitens der gesetzlichen Unfallversicherungsträger übernommen. Im Rahmen einer Wiedererkrankung werden die Kosten der physiotherapeutischen Behandlung jedoch nicht als Dauertherapie erstattet. Ärztlicherseits wird bei der Verordnung einer solchen häufig die Begründung angegeben, dass hierdurch eine Verschlechterung der Funktion verhindert bzw. das aktuelle Belastungs- und Bewegungsausmaß erhalten werden sollen. Studienmäßig ist jedoch nicht belegt, dass eine Dauerphysiotherapie dies bewirken kann. $\mathrm{Zu}$ Recht wird darum seitens der Kostenträger eine solche in der Einzelfallprüfung in der Regel abgelehnt, da dem Patienten zugemutet werden kann, dass er Eigenübungen durchführt oder sich einer Behindertensportgruppe anschließt, wobei diese Kosten regelhaft übernommen werden.

Die Kostenübernahme einer Kryotherapie, einer Ultraschalltherapie, einer Low-Level-Laser-Therapie oder einer elektromagnetischen Feldtherapie kann wegen fehlender oder nicht nachgewiesener Wirksamkeit dem gesetzlichen Unfallversicherungsträger nicht empfohlen werden.

Eine Einlagenversorgung, Schuhzurichtung oder ggf. Brace-Verordnung sind wirksam und werden daher auch seitens der Unfallversicherungsträger übernommen.

Die Kostenübernahme einer TENSTherapie wird im Rahmen der Einzelfallprüfung durch die gesetzlichen Unfallversicherungsträger geprüft. Bei Vorliegen eines chronischen Schmerzsyndroms, insbesondere bei neurologisch begründeten Schmerzen, wird sie regelhaft genehmigt. Die Wirksamkeit ist gesichert. Eine Kostenübernahme bei einer posttraumatischen Arthrose ist dem Kostenträger zu empfehlen, wenn in der Einzelfallprüfung keine ausreichende Schmerzreduktion durch andere Maßnahmen, insbesondere medikamentöse Therapien, erreicht werden kann und eine operative Intervention nicht angezeigt ist.

In zunehmendem Maß wird auch bei der posttraumatischen Arthrose Akupunkturtherapie bei der gesetzlichen Unfallversicherung beantragt. Unter Hinweis auf die obigen Ausführungen ist die Wirksamkeit nicht nachgewiesen. Vielmehr erscheint die Annahme einer Nichtwirksamkeit begründet zu sein. Aufgrund der aktuellen Evidenzlage kann daher die Kostenübernahme für eine Akupunkturtherapie nicht empfohlen werden.

Eine medikamentöse Therapie mit Analgetika, nichtsteroidalen Antiphlogistika und Opioiden ist wirksam und wird auch kostenmäßig durch die gesetzlichen Unfallversicherungsträger übernommen. Die Verordnung von Glukosaminen stellt in der täglichen Praxis kein Problem dar, weil sie selten eintritt. Aufgrund der Evidenzlage ist dann im Einzelfall zu überprüfen, ob es sich um eine Rotta-Präparation handelt, da nur diese wirksam zu sein scheint. Sie kann eine Schmerzlinde- rung, aber keine Funktionsverbesserung bewirken.

Die intraartikuläre Injektion von Kortikoiden stellt eine häufig geübte Therapieform dar. Unter Hinweis auf die EULAR-Empfehlung ist sie mit lang wirkenden Kortikosteroiden bei extremen Schmerzen indiziert, besonders, wenn gleichzeitig ein Erguss besteht. Es liegt jedoch keine ausreichende Evidenz zur Frage der wiederholten Kortisoninjektionen vor. Hier ist im Einzelfall zu prüfen, ob eine wiederholte Kortisoninjektionstherapie zweckmäßig ist oder ob andere Therapieoptionen angezeigt sind.

Im Rundschreiben 106/2004 der Landesverbände der Berufsgenossenschaften wird ausgeführt, dass aufgrund der derzeit bestehenden Datenlage davon ausgegangen werden muss, dass bei einer intraartikulären Injektion von Hyaluronsäure ein geringer positiver Effekt vorhanden sein kann, ohne dass ein eindeutiger Wirksamkeitsnachweis nach unfallbedingten Arthrosen vorliegt. Es wird auf das Risiko einer Gelenkinfektion hingewiesen und dass das Nutzen-Risiko-Verhältnis abgewogen werden müsse. Die Entscheidung zur Kostenübernahme könne nur nach vorheriger kritischer Prüfung im Einzelfall getroffen werden. Der Arbeitskreis würde deshalb nach Einschaltung des beratenden Arztes eine Kostenübernahme nahezu ausschließlich ablehnen. Dies ist auch die bisher geübte Praxis der gesetzlichen Unfallversicherungsträger. Das Rundschreiben der LVBG aus 2004 hat für die einzelnen Unfallversicherungsträger nur Empfehlungscharakter. Diese Empfehlung basiert aber nicht mehr auf dem aktuellen wissenschaftlichen Evidenzstand. Die Cochrane-Übersichtsartikel aus dem Jahr 2006 und das systematische Review von 5 Metaanalysen belegen die Wirksamkeit von Hyaluronsäureinjektionen [4, 17]. Unter dem Hinweis auf die EULAR-Empfehlung sollten die dort angegebenen nichtmedikamentösen Therapien sowie die medikamentösen lokalen und oralen Behandlungsmöglichkeiten zuvor ausgeschöpft worden sein. Sollten diese Behandlungsmaßnahmen nicht mehr ausreichen, sollte seitens der Unfallversicherungsträger in der Einzelfallprüfung und nach Abwägung des Nutzen-Risiko-Verhältnisses die Kostenübernahme 
überprüft werden. Bis zu einer geplanten Überprüfung durch den gesetzlichen Unfallversicherungsträger bzw. die Landesverbände der BG hat das Rundschreiben 106/04 mit seinem Empfehlungscharakter Bestand. Eine Überprüfung durch die im Arbeitskreis vertretenen Verwaltungen ist aufgrund der aktuellen wissenschaftlichen Evidenz erforderlich.

\section{Fazit}

Ein Vergütungsanspruch des Arztes ist nur dann gegeben, wenn die jeweilige ärztliche Leistung medizinisch erforderlich und zweckmäßig ist. Behandlungsmaßnahmen, die diese Voraussetzung eindeutig nicht erfüllen, sind seitens der Unfallversicherungsträger nicht zu honorieren. Der Arzt ist über die Gründe der Rechnungskürzung zu informieren. Unter Hinweis auf die Erläuterungen zum Abkommen der Ärzte und Unfallversicherungsträger fallen Streitfälle über die Honorierung der ärztlichen Leistung nicht unter den Schiedsvertrag. Bei Streitfällen zwischen dem behandelnden Arzt und dem gesetzlichen Unfallversicherungsträger sind die Gerichte der Sozialgerichtsbarkeit zuständig. Dem behandelnden Arzt ist es unbenommen, gegen eine ablehnende Entscheidung zur Kostenübernahme von Behandlungsmaßnahmen im Einzelfall Widerspruch zu erheben. Hierbei sollte jedoch nicht auf die Therapiefreiheit und die persönliche Erfahrung verwiesen werden, stattdessen sollte die Begründung den Kriterien der evidenzbasierten Medizin entsprechen.

\section{Korrespondenzadresse \\ Prof. Dr. T. Tiling}

Klinik für Unfallchirurgie, Lehrstuhl für Unfallchirurgie/Orthopädie der Universität Witten/ Herdecke, Krankenhaus Merheim, Kliniken der Stadt Köln

Ostmerheimer Straße 200, 51109 Köln thomas@tiling.de

Interessenkonflikt. Der korrespondierende Autor weist auf folgende Beziehung/en hin: Der Erstautor ist beratender Arzt der Maschinenbauund Metall BG, beratender Arzt des Landesverbandes NRW der gesetzlichen Unfallversicherungsträger und Referent der Deutschen Gesetzlichen Unfallversicherung, Rechnungsprüfung, und erhält für seine Tätigkeit für die BG bzw. Deutsche Gesetzliche Unfallversicherung ein Honorar.

\section{Literatur}

1. Bellamy N, Campbell J, Robinson V et al. (2006) Intraarticular corticosteroid for treatment of osteoarthritis of the knee. Cochrane Database Syst Rev 2: CD005328

2. Bellamy N, Campbell J, Robinson V et al. (2006) Viscosupplementation for the treatment of osteoarthritis of the knee. Cochrane Database Syst Rev 2: CD005321

3. Bennell KL, Hinman S, Metcalf BR et al. (2005) Efficacy of physiotherapy management of knee joint osteoarthritis: a randomised, double blind, placebo controlled trial. Ann Rheum Dis 64: 906-12

4. Bereiter-Hahn J (2006) Mertens Gesetz und Recht. Schmidt, Berlin, Erg-LFG bis 1/07

5. Biswal S, Medhi B, Pandhi P (2006) Longterm efficacy of topical nonsteroidal antiinflammatory drugs in knee osteoarthritis: metanalysis of randomized placebo controlled clinical trials. J Rheumatol 33: 1841-1844

6. Brosseau L, MacLeay L, Robinson V et al. (2003) Intensitiy of exercise for the treatment of osteoarthritis. Cochrane Database Syst Rev 2: CD004259

7. Brosseau L, Yonge KA, Robinson V et al. (2003) Thermotherapy for treatment of osteoarthritis. Cochrane Database Syst Rev 4: CD004522

8. Brosseau L, Welch V, Wells G et al. (2004) Low level laser therapy (classes I, II and III) for treating osteoarthritis. Cochrane Database Syst Rev 3: CD002046

9. Brouwer RW, Yakma TS, Verhagen AP et al. (2005) Braces and orthoses for treating osteoarthritis of the knee. Cochrane Database Syst Rev 1: CD004020

10. Buszewicz M, Rait G, Griffin M et al. (2006) Self management of arthritis in primary care: randomised controlled trial. BMJ 333: 867-868

11. Casimiro L, Barnsley L, Brosseau L et al. (2005) Acupuncture and electroacupuncture for the treatment of rheumatoid arthritis. Cochrane Database Syst Rev 4: CD003788

12. Cepeda MS, Camargo F, Zea C et al. (2006) Tramadol for osteoarthritis. Cochrane Database Syst Rev 3: CD005522

13. Chodosh J, Morton SC, Mojica W et al. (2005) Meta-analysis: chronic disease self-management programs for older adults. Ann Intern Med 143: 427438

14. Deutsche Gesellschaft für Orthopädie und orthopädische Chirurgie und Berufsverband der Ärzte für Orthopädie (Hrsg) (2002) Leitlinien der Orthopädie: Gonarthrose, 2. Aufl. Deutscher Ärzteverlag, Köln

15. Deutsche Gesetzliche Unfallversicherung (2006) Hinweise der Unfallversicherungsträger zur Bearbeitung von Arztrechnungen des Arbeitskreises "Rechnungsprüfung". Deutsche Gesetzliche Unfallversicherung, Sankt Augustin

16. Devos-Comby L, Cronan T, Roesch SC (2006) Do exercise and self-management interventions benefit patients with osteoarthritis of the knee? A metaanalytic review. J Rheumatol 33: 744-756

17. Divine JG, Zazulak BT, Hewett TE (2006) Viscosupplementation for knee osteoarthritis. Clin Orthop 455: 113-122

18. Ezzo J, Hadhazy V, Birch S et al. (2001) Acupuncture for osteoarthritis of the knee: a systematic review. Arthritis Rheum 44: 819-825

19. Fransen M, McConnell S, Bell M (2001) Exercise for osteoarthritis of the hip or knee. Cochrane Database Syst Rev 2: CD004286
20. Garner SE, Fidan DD, Frandish RR et al. (2005) Rofecoxib for osteoarthritis. Cochrane Database Syst Rev 1: CD005115

21. Hulme J, Robinson V, DeBie R et al. (2002) Electromagnetic fields for the treatment of osteoarthritis Cochrane Database Syst Rev 1: CD003523

22. Jordan KM, Arden NK, Doherty M et al. (2003) EULAR Recommendations 2003: an evidence based approach to the management of knee osteoarthritis. Report of a task force of the Standing Committee for International Clinical Studies including Therapeutic Trials (ESCISIT). Ann Rheum Dis 62: 11451155

23. Lauterbach $\otimes(2005)$ UV (SGB VII), 4. Aufl. 27. Lfg. 囚

24. Mazzuca S A, Brandt KD, Katz BP et al. (2004) Can a nurse-directed intervention reduce the exposure of patients with knee osteoarthritis to nonsteroidal antiinflammatory drugs? J Clin Rheumatol 10: 315-322

25. McCarthy CJ, Callaghan MJ, Oldham JA (2006) Pulsed electromagnetic energy treatment offers no clinical benefit in reducing the pain of knee osteoarthrits: a systematic review. BMC Musculoskelet Disord 7: 51

26. Noeske B, Hamacher B, Franz J (1997) Erläuterungen zum Abkommen Ärzte/Unfallversicherungsträger. Dühringshofen, Berlin

27. Osiri M, Welch V, Brosseau L et al. (2000) Transcutaneous electrical nerve stimulation for knee osteoarthritis. Cochrane Database Syst Rev 4: CD002823

28. Robinson VA, Brosseau L, Peterson J et al. (2001) Therapeutic ultrasound for osteoarthritis of the knee. Cochrane Database Syst Rev 3: CD003132

29. Rubin BR (2005) Management of osteoarthritic knee pain. J Am Osteopath Assoc [Suppl] 105: 2328

30. Sackett DL (2000) Protection for human subjects in medical research. JAMA 283: 2388-2389

31. Scharf HP, Mansmann U, Streitberger K et al. (2006) Acupuncture and knee osteoarthritis: a three-armed randomized trial. Ann Intern Med 145: 12-20

32. Towheed TE (2006) Pennsaid therapy for osteoarthritis of the knee: a systematic review and metaanalysis of randomized controlled trials. J Rheumatol 33: 567-573

33. Towheeed TE, Maxwell $L$, Anastassiades TP et al. (2005) Glucosamine therapy for treating osteoarthritis. Cochrane Database Syst Rev 2: CD002946

34. Towheed TE, Maxwell L, Judd MG et al. (2006) Acetaminophen for osteoarthritis. Cochrane Database Syst Rev 1: CD004257

35. Tugwell PS, Wells GA, Shainhouse JZ (2004) Equivalence study of a topical diclofenac solution for relieving symptoms of primary osteoarthritis of the knee: a randomized controlled trial. J Rheumatol 31: 2002-2012

36. Watson M, Brookes ST, Kirwan JR et al. (2006) Nonaspirin, non-steroidal anti-inflammatory drugs for treating osteoarthritis of the knee. Cochrane Database Syst Rev 1: CD000142 